\title{
A SYSTEMATIC REVIEW ON SELF-MICRO EMULSIFYING DRUG DELIVERY SYSTEMS: A POTENTIAL STRATEGY FOR DRUGS WITH POOR ORAL BIOAVAILABILITY
}

\author{
G. V. RADHA ${ }^{1 *}$, K. TRIDEVA SASTRI ${ }^{1}$, SADHANA BURADA ${ }^{1}$, JAMPALA RAJKUMAR1
}

${ }^{1}$ GITAM Institute of Pharmacy, GITAM Deemed to be University, Rushikonda, Visakhapatnam, Andhra Pradesh State, India Email: radhagadela@gmail.com

Received: 26 Sep 2018, Revised and Accepted: 19 Nov 2018

\begin{abstract}
Currently a marked interest in developing lipid-based formulations to deliver lipophilic compounds. Self-emulsifying system has emerged as a dynamic strategy for delivering poorly water-soluble compounds. These systems can embrace a wide variety of oils, surfactants, and co-solvents. An immediate fine emulsion is obtained on exposure to water/gastro-intestinal fluids. The principal interest is to develop a robust formula for biopharmaceutical challenging drug molecules. Starting with a brief classification system, this review signifies diverse mechanisms concerning lipidbased excipients besides their role in influencing bioavailability, furthermore pertaining to their structured formulation aspects. Consecutive steps are vital in developing lipid-based systems for biopharmaceutical challenging actives. Such a crucial structured development is critical for achieving an optimum formula. Hence lipid excipients are initially scrutinized for their solubility and phase behavior, along with biological effects. Blends are screened by means of simple dilution test and are consequently studied with more advanced biopharmaceutical tests. After discerning of the principle formula, diverse technologies are offered to incorporate the fill-mass either in soft/hard gelatin capsules. There is also feasibility to formulated lipid-system as a solid dosage form. Although such solid technologies are desirable but such should not undermine the biopharmaceutical potential of lipid-formulations.
\end{abstract}

Keywords: Absorption, Lipid-based excipients, Lymphatic uptake, Precipitation, SMEDDS, Bioavailability

(C) 2019 The Authors. Published by Innovare Academic Sciences Pvt Ltd. This is an open access article under the CC BY license (http://creativecommons.org/licenses/by/4.0/) DOI: http://dx.doi.org/10.22159/ijap.2019v11i1.29978

\section{INTRODUCTION}

With today's contemporary drug discovery techniques, innovating drug candidates without compromising on safety and efficacy is a challenge. Though there has been a remarkable success in the development of new drug candidates, there are still unmet needs in healthcare which need effective therapy [1]. Predominantly new chemical entities (NCE) (about 40\%) with potential therapeutic activity and other pharmacokinetic aspects are compromised as a result of poor solubility, concerns with permeability, rapid metabolism and early elimination. Therefore, the pharmaceutical drug discovery phase for an NCE with appropriate biopharmaceutical properties is crucial for the successful development of the new drug.

Hence the significance of pharmaceutical technology emerged to formulate bio pharmaceutically competent drugs. Several strategies exist to deal with drug molecules with lipophilicity or permeability includes micronization of crystalline solids, amorphous formulation, and lipid-based formulations. Lipid-based formulations, especially self-emulsifying formulations; self-micro emulsifying drug delivery system or self-nano emulsifying drug delivery system (SMEDDS or SNEDDS) prominently gained great significance as a successful approach for poorly soluble drugs amid other approaches [2-4]. Liposomes, lipoplexes, solid-lipid nanoparticle (SLN), SMEDDS, microemulsion, and macroemulsions mostly contribute to lipidbased formulations. In recent years, lipid-based formulation attained importance with significant emphasis on self-emulsifying drug delivery system (SEDDS) [5]. Lipid-based formulations, especially SEDDS are emulsions with characteristically fine globule size and possess greater thermodynamic stability [6-8].

In gastrointestinal tract (GIT) upon mild agitation or gastrointestinal motility, SEDDS composed of isotropic mixtures of oils, surfactants, and co-solvents produce rapid fine emulsions [9, 10]. SEDDS effectively produce emulsions with a droplet size range of 100-200 nm for SMEDDS whereas SNEDDS produce less than $100 \mathrm{~nm}$.

It's significant to note that approach for producing fine emulsion by SMEDDS/SNEDDS is analogous to low-energy emulsification technique for nanoemulsions. The concentration of the surfactants and cosurfactant within SMEDDS system influence diffusion of the drug from oil globule to dissolution media, therefore, diffusion fundamentally limits the rate of dissolution $[11,12]$. The major difference between SEDDS, SMEDDS, and SNEDDS are listed in (table 1).

Table 1: Major difference between SEDDS, SMEDDS, and SNEDDS

\begin{tabular}{|c|c|c|c|}
\hline Property & SEDDS & SMEDDS & SNEDDS \\
\hline Size & $>300 \mathrm{~nm}$ & $<250 \mathrm{~nm}$ & $<100 \mathrm{~nm}$ \\
\hline Appearance & Turbid & Optically clear & Optically clear \\
\hline Hydrophilic-lipophilic balance (HLB) value & $<12$ & $>12$ & $>12$ \\
\hline Classification as per LFCS & Type II & Type IIIB & Type IIIB \\
\hline Concentration of oil & $40-80 \%$ & $>20 \%$ & $>20 \%$ \\
\hline Concentration of surfactant & $30-40 \%$ & $40-80 \%$ & $40-80 \%$ \\
\hline
\end{tabular}

Colin Pouton categorically classified lipid-based formulations due to their diversity. The lipid formulation classification system (LFCS) categorized into four categories (table 2) [13]. All categories include formulations with isotropic systems, usually of mixtures but also consist of single excipient e. g. oil. Type I lipid-based drug delivery system (LBDDS) include only triglyceride oil or with certain mixtures with its glycerides. Formulations belonging to this category potentially do not self-disperse, it requires bile salts, lipolysis products and also phospholipids to lower interfacial tension post administration [14]. Other categories, unlike the type I requires only a gentle agitation that spontaneously yields a fine emulsion. Such formulations additionally include surfactants along with oil components. Based on the nature of 
surfactants employed, LFCS type II category differs from type IIIA. Where the type II system includes one or more lipophilic surfactant, and type IIIA mostly consist more hydrophilic. The latter comprises of oil mixtures, hydrophilic surfactant, and co-solvents. Moreover, type
IIIB systems comparatively consist lesser concentration of oil and include higher concentrations of hydrophilic surfactants with more cosolvents. Finally pertaining to category IV consists of no amount of oil, typically include surfactants and co-solvents only.

Table 2: the Lipid-based classification system

\begin{tabular}{|c|c|c|c|c|c|}
\hline \multirow[t]{2}{*}{ Excipients } & \multicolumn{5}{|c|}{ The content of formulation $(\%, w / w)$} \\
\hline & Type I & Type II & Type IIIA & Type IIIB & Type IV \\
\hline Oils: triglycerides mixed mono and diglycerides & 100 & $40-80$ & $40-80$ & $<20$ & - \\
\hline Surfactants $(\mathrm{HLB}<12)$ & - & $20-60$ & - & - & $0-20$ \\
\hline Surfactants (HLB $>12)$ & - & - & $20-40$ & $20-50$ & $30-80$ \\
\hline Hydrophilic co-solvents (e. g. PEG etc) & - & - & $0-40$ & $20-50$ & $0-50$ \\
\hline
\end{tabular}

The efficiency of the LBDDS builds in the right from the selection of suitable vehicle composition, and coherent design for the significant drug candidate. Basically, for effective enhancement of oral absorption, the system needs to improve the rate and extent of dissolution and potentially should maintain the drug in the solubilized form in the GIT. The potential advantages extended by lipid-based formulation system are compromised in case of precipitation of drug occurs from the system. The current article emphasizes significant aspects of lipidbased formulation and their characterization.

\section{Advantages of smedds}

a) Storage: Compared to other emulsions SMEDDS are potentially better for enabling solubility of lipophilic drugs. On storage creaming over time is a concern for macroemulsions, unlike macroemulsions SMEDDS are thermodynamically stable facilitating easy storage [15].

b) Stability: As water not being a significant component in formulation systems pertaining to SMEDDS, in contrast to other emulsions possess enhanced chemical and physical stability on longterm storage [16].

c) Compliance and palatability: Majority of SMEDDS are effectively formulated into capsules or tablets dosage forms, enables effortless administration moreover occupies lesser volume thereby resolving palatability concerns and enhancing patient compliance [17].

d) Food effects: Fate of absorption is not impeded by the presence of food, rather lipophilic contents aid in the absorption of the drug from the formulation systems [18].

e) Quick onset of action: SMEDDS prominently expedite spontaneous and rapid oral absorption of the active, significantly result in the quick onset of action [19].

f) Simple manufacturing and scale-up: Comparatively employing simpler and economical manufacturing facilities it's easier to manufacture SMEDDS at large scale [20].

\section{Limitations of smedds}

SMEDDS formulations significantly possess various advantages, albeit inevitable limitations associated with the formulation system;

a) Drug precipitation: during dilution in gastrointestinal fluids, active undergo precipitation from the formulation system. It is necessary for the formulation system to maintain the drug in the solubilized state in GIT effectively, precipitation of active yields negative effects and compromises the advantages extended by the lipid-based formulation system.

b) The dilution effect of lipophobic solvents increases precipitation propensity of the drug on dilution. Thus, consequentially requires incorporation of polymers to alleviate drug precipitation in vivo [21].

c) Encapsulation in gelatin capsules: Certain demerits allied with gelatin capsules, volatile co-solvents incorporated in the formula tend to migrate to shells consequentially lipophilic drugs are precipitated [22].

d) Storage: liquid SMEDDS show difficulty in storage and handling besides stability concerns. e) Correlation between lymphatic transport and lipophilicity in addition to triglyceride solubility needs to be perceived with more precise predictive model [23].

f) An absence of significant predictive in vitro models for characterising formulation systems [24].

g) Due to the presence of unsaturated fatty acids, lipid excipients are susceptible to oxidation; explicit process control is required to restrict polymorphic alterations of excipient matrix [25].

\section{Drug absorption}

Lipid-based formulation systems are derived with a blend of three major excipients, which include oil, surfactant, and co-surfactant. These formulations characteristically enhance with rapid dissolution thus improve drug absorption from GIT, enabling the formation of solubilised phases by altering particle size to almost molecular state, giving rise to solid-state solution in the formulation system, altering drug uptake, efflux and deposition besides modifying transportation associated with enterocytes and also enriching drug transport through intestinal lymphatic system to systemic circulation [26-28].

\section{Lymphatic system}

Lymphatic system plays a significant role with its considerable network throughout the body in transporting the drug to the systemic circulation. Lymphatic transport of drug surpasses the first-pass metabolism and also aids in targeting certain disease conditions associated with the lymphatics, like lymphomas. The promising mechanism includes (I) facilitating transcellular absorption as a result of improved membrane fluidity, (II) opening of tight junctions enabling paracellular transport, (III) inhibition of P-glycoprotein (p-gp) and CYP450 yielding to enhanced intracellular concentration and also increased surfactants residence time, (IV) stimulating lipoprotein/chylomicron production [29].

\section{Digestion and solubilization}

The equilibrium among the solubility of the drug in the aqueous phase of the GIT and permeation over the lipophilic membrane govern its rate and extent of absorption [30]. Gastric lipase instigates the digestion of triglycerides to post oral ingestion of the lipid-based formulations. Crude emulsion is formed from the mechanical mixing of the stomach. Triglyceride is further breaking down to di-and monoglyceride in the small intestine and fatty acid by pancreatic lipase along with its co-factor co-lipase203, acting predominantly at sn- 1 and sn-3 positions of triglyceride to yield 2monoglyceride and free fatty acid [31].

The sn-2 position of phospholipids is hydrolysed to lysophosphatidylcholine and fatty acids by pancreatic phospholipase A2 and exogenous lipids present in small intestine trigger secretion of endogenous lipids from the gallbladder, comprising phospholipids, bile salts, and cholesterol [32]. Formerly formed monoglycerides, lysophospholipids, and fatty acids are consequently combined into a series of colloidal structures along with micelles and vesicles (uni/multilamellar) in existence of bile salts. These formed metabolites significantly enhance solubilization and absorption potential of the small intestine for digested lipids and subsequent drug.

\section{Effects of lipid-based excipients}

Oral dosage forms significant in vivo performance is pronounced by the solubility of the lipophilic drug. Since drug mostly remains in the 
solubilized state in LBDDS, as a result, the dissolution step is circumvented. This gives the vital advantage for delivery of lipophilic drugs, but this phenomenon is inadequate if the solubilized state is lost upon aqueous dilution and dispersion. If the active precipitates, it is believed that the rate of re-dissolution is low compared to intestinal transit time. Thus, such precipitation results in poor drug absorption. However always precipitation doesn't initiate inconsistent drug absorption. In certain occurrences in which precipitate has time for re-dissolution. Drug solubility and also the amount of drug precipitate in comparison with dose influence the kinetics $[33,34]$. It is observed precipitation in the amorphous state is positively less critical for re-dissolution compared to the crystalline state.

Preferably, LBDDS efficiently delivers a drug in solubilised form and sustain ample solubilisation throughout the gastrointestinal transit. Lipid-based excipients proficiently enhance drug solubility and can promote supersaturation that is adequate for drug absorption. Besides the effects of solubility and drug release, the formulation system further promotes by additional mechanisms that potentially influence oral bioavailability. Most of the lipid-based excipients like fatty acids, glycerides and surfactants are inherently enhance permeability. This might be resultant of altered membrane fluidity or tight junctions. Alternatively, excipients enhance permeability when they effectively interact with efflux transporters. Most familiar efflux transporter in the human intestine e. g. p-gp. Once the efflux pump is inhibited by lipid excipients substrates are believed to observe enhanced permeation. Most of the polysorbates, polyethylene glycols, medium-chain glycerides co-polymers of pluronics exhibit inhibiting properties on efflux transporters. Surfactants possessing amphiphilic structure precisely inhibit p-gp. Furthermore, amphiphilic surfactants influence other efflux transporters like breast cancer resistance protein (BCRP) [35].

Mostly lipid-based excipient modifies drug absorption by various mechanisms. Majority of the surfactants that affect efflux transporters usually affect drug solubilisation. Excipients certain times are capable of influencing cytochrome P450 metabolism of drug besides p-gp transporter in the intestine. In conclusion to certain aforesaid mechanisms, surfactants show additional simulate lipoproteins secretion in intestine e. g. chylomicrons. Drugs transported through the lymphatic pathway are benefited with the altered chylomicron synthesis. All these contemplations deliberately exhibit that excipients in a formulation system are essentially several functions. As several mechanisms are associated with formulation system, it becomes challenging to predict and understand the biopharmaceutical effects of the formulation system. Predominantly formulation systems yield beneficial effects on bioavailability of lipophilic drugs. In the case of soluble molecules desire a protective outcome from enzymatic or chemical degradation in the formulation system.

\section{Mechanism of self-emulsification}

Reiss recommended that self-emulsification occurs when an entropy change intended for dispersion is superior over the energy essential to upsurge the surface area of the dispersion [36]. In a typical emulsion formation, the free energy is a direct function of the energy essential to yield new surface amid two phases and is understood by the given equation.

$$
\Delta \mathrm{G}=\sum \mathrm{N}_{\mathrm{i}} \pi \mathrm{r}_{\mathrm{i}}^{2} \sigma
$$

Where $\Delta \mathrm{G}$ denotes free energy related to the process (overlooking the free energy of mixing), $N$ denotes the number of droplets, $r$ is the radius of the formed droplet and $\sigma$ represents interfacial energy with time.

In order to lower the interfacial area, the two phases of the emulsion favour separation with the time, giving rise to the free energy of the systems. Stabilization of emulsion employing typical emulsifying agents posts aqueous dilution by producing a monolayer around the emulsion droplets, thereby lowering the interfacial energy further preventing coalescence by forming a barrier. Unlike typical, selfemulsifying systems potentially emulsify spontaneously since free energy essential to produce the emulsion is very low beside positive or negative [37].
To emulsify it is essential for the interfacial structure to remain immune to surface shearing since emulsification necessitates slight input energy, presume destabilization by shrinking local interfacial zones. Through significant water penetration into several liquid crystals and other structural components present on the droplet's surface facilitate better emulsification [38, 39].

By the incorporation of a binary blend (oil/surfactant) to water yields an interface between oil and aqueous phases. As a consequence of aqueous penetration through the interface water is solubilised within oil phase. This process of solubilisation takes place until the limit is reached near to the interphase [40]. Furthermore, the dispersed liquid crystal phase is formed as an outcome of aqueous penetration. Ultimately resulting in liquid crystal in the vicinity with the interface, the surfactant concentration in the binary mixture governs the true amount. Thereby with moderate agitation water expeditiously penetrate into aqueous cores and promote interface disruption including droplet formation. Due to development of liquid crystal interface neighboring the oil droplets, stability in terms of coalescence is imparted to the formulation system.

\section{Rationale for selection of smedds components}

Characteristically SMEDDS formulation includes drug, oil, surfactant, and co-surfactant;

\section{Drug}

For the development of SMEDDS formulation, the lipophilicity along with the dose of the active should be thoroughly scrutinized. Preferably, the drug should possess low dose; $\log p \geq 2$ moreover should not be susceptible to the first-pass metabolism. The drug inherently should exhibit significant solubility in pharmaceutically acknowledged lipids, surfactants and co-solvents [41].

Oils

Medium chain triglycerides possessing carbon atoms ranging from 6 and 12 are fundamentally transported by the portal blood to the systemic circulation. However long-chain triglycerides possessing carbon atoms higher than 12 are transported through intestinal lymphatics. Since medium chain triglycerides prominently have high solvent capacity moreover not susceptible to oxidation, they are potentially employed in lipid-based formulation systems [42, 43].

\section{Surfactants}

Surfactants contribute the interfacial film besides the interfacial tension being greatly reduced to a low value which enables the dispersion process. HLB value and concentration of surfactant are significant in the selection of surfactant. For an efficient performance, the emulsifier employed in the system should possess HLB value higher than 12 , which promote the formation of small $\mathrm{o} / \mathrm{w}$ droplets with expeditious dispersion in aqueous media. Basically, non-ionic surfactant with greater HLB value is preferred for the formulation system as they are less toxic than ionic surfactants [44].

\section{Co-surfactants}

Flexibility to the interfacial layer is imparted by co-surfactants where the interfacial tension is greatly reduced to an almost negative value. The flexibility induces various curvatures essential to form micro emulsion across a wide range. As a co-surfactant mostly, medium chain length alcohols are employed.

\section{Role of excipient selection on bioavailability}

A predominantly broad range of excipients are available for formulating lipophilic drugs in lipid formulations. Conventionally lipidbased systems include excipients subsequently: 1) lipids: natural or synthetic lipids; 2) surfactants: hydrophilic or hydrophobic non-ionic surfactants; 3) hydrophilic solvents: to enhance dispersion and for better solvent capacity; 4) co-solvents: significantly lower viscosity of the system and to enable dispersion. From formulation viewpoint, the excipient choice will influence dispersion properties, drug load, drug solubilization and significantly stability. Moreover, pharmacokinetic characteristics of excipients are also considered which may significantly affect the bioavailability. 


\section{Lipids}

The fundamental role of lipids is to solubilise drug and further sustain it in the solubilised state during the GI-tract transit. In case of drug precipitation as an outcome of lipid digestion, the drug must be resolubilized in intestinal fluids for being absorbed. This is essential specifically for the biopharmaceutical classification system (BCS) class II and IV [45]. For formulating lipid-based systems, diverse lipid excipients are existing like short, medium and longchain triglycerides. Basically, medium chained triglycerides are digested at a faster rate compared to long chained triglycerides and also former is digested completely. Furthermore because of the high solvent capacity drug to a greater extent is kept in a solubilised state in medium chained triglycerides. Whereas due to poor solvent capacity short chain triglycerides yields extensive precipitation of drug. Moreover, apart from the aforementioned lipid digestion aspects, considerably lipid influence the absorption profiles of the drug. For instance, portal vein absorption via liver is predominant in medium chain triglycerides and lymphatic absorption is bypassing the first-pass metabolism in case of long-chain triglycerides.

\section{Surfactants}

Extremely studied excipients in the class of non-ionic are commonly include cremophor EL and cremophor RH40. Albeit cremophor EL and cremophor RH40 belong to the same family compared to cremophor RH40, cremophor EL possess a lower degree of ethoxylation besides being unsaturated. In vivo digestion aspects should be scrutinized for their inclusion in the formulation. Concerning to the surfactants cremophor EL is more vulnerable to digestion when compared to cremophor RH40 [46]. The explanations for varied digestions are still unclear; however it has been attributed to variances with respect to reactivities of saturated castor oil glyceride backbone associated with cremophor RH40 yields diverse reaction products compared to cremophor EL. Cremophor RH40 being less prone digestion significantly optimum for maintaining the drug in the dissolved state for an extended period when compared to cremophor EL [47]. Although as aforementioned concepts are considerable under in vivo conditions, the comparative affinity of lipases towards excipients will influence the extent of digestion and drug solubilization, signifying the importance formulation optimization. Additionally, low lipid concentrations increase the susceptibility of drug precipitation as a result of dilution which ultimately fails to keep the drug in the dissolved state. This clearly implies the inevitability to optimize the ratio of lipid to surfactant for improved bioavailability. Besides the high concentration of surfactants results in micelle formation which may hamper the absorption process as it increases the molecular weight of the entire complex. And also, prolonged use of surfactants is not advisable as they potentially disrupt cell membrane, toxicity and adverse effects [48].

\section{Hydrophilic solvents}

LFCS type III formulations require minor quantities of hydrophilic solvents like triethyl citrate, propylene glycol and polyethylene glycol 400 (PEG-400) are employed to facilitate emulsification through dispersion and stranding [49]. Apart from excipient toxicities handful of hydrophilic solvents possess essential pharmacokinetic characteristics. PEG- 400 belonging to this category, when consumed in larger quantities affect the gastric motility and thereby affect drug absorption. As an outcome of poor absorption triggers PEG-400 induced osmotic activity on subsequent water retention results stimulating intestinal motility. For many drug molecules, small intestine predominantly facilitates major absorption due to declined transit time will reduce time offered for absorption of poorly soluble and slowly dissolving molecules. Nevertheless, PEG-400 at lower concentrations prominently enhance the absorption of certain drug molecules like ranitidine, due to modified intestinal permeability [50, 51]. Albeit only small quantities of hydrophilic solvents are employed in lipid-based systems, aforementioned pharmacokinetic impact should be considered while excipient selection.

\section{Cosolvents}

Basically, minimal concentrations of cosolvents like ethanol are used to lower viscosity besides assist in the dispersion process. At such low concentrations, the cosolvents are pharmacokinetically insignificant.

\section{Development of formulation \\ Excipient selection for scrutinizing drug solubility}

To begin with the formulation development of lipophilic molecules comprehensive pharmaceutical profiling is performed. Stability and solubility tests should be accompanied beside biopharmaceutical assessment. Initial impression with drug absorption barrier is learned by considering the BCS class, the maximum absorbable dose [52-54]. With recent advancements like physiologically based pharmacokinetic modelling (PBPK) enable drug evaluations simpler [55-58]. Due to uncertain input parameters, such models are inadequate predictability at initial stages of the development. Nevertheless, PBPK modelling appreciably predict the sensitivity of drug absorption considering diverse factors. Specifically, dose fraction absorbed is significant in view of formulation parameters. In such instance's formulation can be developed deliberately using parameter sensitivity analysis [59]. Such strategies are considered in SMEDDS or SEDDS.

The formulation system should suitably balance the dose with drug solubility in oils. Technically preformulation data initially comprises drug solubility in medium and long chain triglycerides. The obtained solubility measures describe how accurately the desired dose strength is achieved. Certainly, in an optimized blend like SMEDDS drug solubility is extremely enhanced but there are certain demerits associated with the increase in solubility. Few drugs are poorly water solubility as a result of their lipophilicity. Such molecules possess higher value about $2-4$ of the partition coefficient $(\log \mathrm{P})$, are usually suitable candidates for lipid-based systems. For other molecules, the limitation of aqueous solubility instigates from high crystal energy. The solubility impediment as an outcome of solid-state properties is most prominent with molecules possessing moderate to low lipophilicity [60]. For aforesaid compounds, it is mostly tough in identifying oil vehicles having an adequate solvent capacity. Therefore, such molecules should be avoided for lipid-based systems and looked for alternate formulation systems like solid dispersions etc.

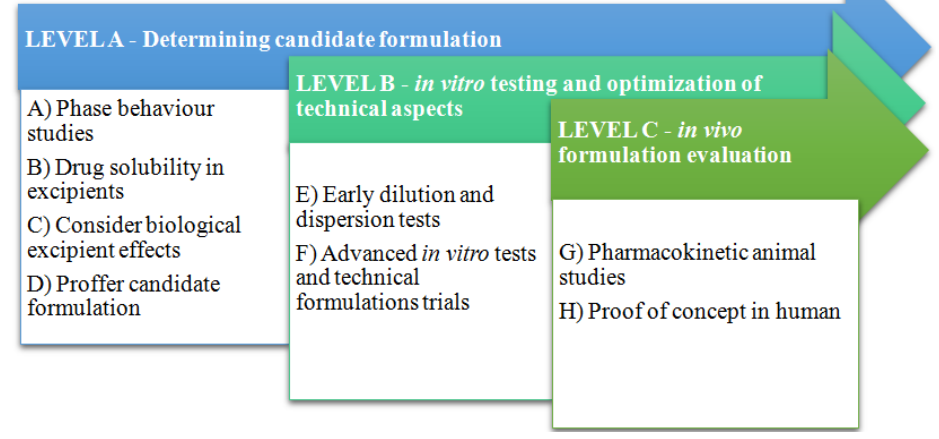

Fig. 1: Structured approach for explicating ideal excipients additionally development of a lipid-based system [63] 
Table 3: Major solubilising excipients employed in commercially available lipid-based systems

\begin{tabular}{|c|c|c|c|}
\hline Water-soluble excipients & Triglycerides & Surfactants & References \\
\hline Bees wax & Long-chain triglycerides: & Polysorbate 20 (tween 20) & [64] \\
\hline Oleic acid & Hydrogenated soyabean oil & Polysorbate 80 (tween 80 ) & {$[64]$} \\
\hline Soy fatty acids & Hydrogenated vegetable oil & Sorbitan monolaurate (span 20$)$ & [64] \\
\hline D- $\alpha$-Tocopherol (vitamin A) & Corn oil, olive oil, & D- $\alpha$-Tocopheryl PEG 1000 succinate (TPGS) & {$[64]$} \\
\hline Corn oil mono-di-triglycerides & soyabean oil, peanut oil, & Glyceryl monooleate & [64] \\
\hline $\begin{array}{l}\text { Medium chain (C8/C10) mono and } \\
\text { di glycerides }\end{array}$ & Sesame oil & Polyoxyl 35 castor oil (cremophor EL) & [64] \\
\hline \multirow[t]{6}{*}{ Propylene glycol esters of fatty acids } & Medium-chain triglycerides: & Polyoxyl 40 hydrogenated castor oil (cremophor RH40) & {$[64]$} \\
\hline & Caprylic/capric & Polyoxyl 60 hydrogenated castor oil (cremophor RH60) & {$[64]$} \\
\hline & $\begin{array}{l}\text { Triglycerides derived from } \\
\text { coconut oil or palm oil }\end{array}$ & PEG 300 oleic glycerides (labrafil ${ }^{\circledR}$ M-1944 CS) & {$[64]$} \\
\hline & & PEG 300 linoleic glycerides (labrafil ${ }^{\circledR}$ M-2125 CS) & {$[64]$} \\
\hline & & PEG 400 caprylic/capric glycerides (labrasol ${ }^{\circledR}$ ) & {$[64]$} \\
\hline & & PEG 1500 lauric glycerides (gelucire ${ }^{\circledR} 44 / 14$ ) & [64] \\
\hline
\end{tabular}

Once confirmed with the scrutiny of new drug candidate suitability for lipid-based systems, it is significant to design formulation development in a structured manner. (fig. 1) exhibits such an approach that can be employed as a formulation development design.

The purpose of level-A is to achieve candidate systems for further screening and evaluation. This begins with the choice of prominent excipients and blends $[61,62]$. Usually, excipients possess diverse functionalities in blends e. g. being an oil, surfactant, co-surfactant or the additive can be a solvent. (table 3) exhibits solubilizing excipients employed in different commercially available for lipidbased systems. Functionality is further tough to assign, as most excipients like gelucire 44/14, are significantly are a complex blend of complex substances. Regardless of the unforeseeable character of any characterization, it is still considered to obtain a better perspective of various formulations.

The oil is a critical component of the system. The selection is made from medium-to long-chain triglycerides with different degrees of saturation. On addition of partially or complete hydrolysed oils enhances dispersion behaviour and may improve the solubility of drug molecules. This characteristic of drug solubility must be addressed at an initial level as shown in (fig. 1). Mostly the preformulation data are accompanied by the screening of drug solubility in a series of several lipid-based excipients [65, 66]. Formulations essentially require excipients possessing high solvent capacity. Distinctive solvents like ethanol etc should be scrutinized in the primary solubility trails. Moreover, all tested excipients should be confirmed with the toxicological and regulatory status. Predominantly important for surfactants as surface actives usually have critical tolerability [67].

TGPS is considered suitable for the formulation being the derivative of vitamin $E$ is well endured and benefits from broad regulatory acceptance. But the excipient grades must be cautiously evaluated concerning regulatory aspects. Based on the resultant excipient precedencies the additive is restricted to both preclinical, and early clinical studies or a universal regulatory acceptance is possible to enable a market formulation. The usage of excipients without compliance with regulatory aspects must be discouraged. Certain times drug properties necessitate excipients that have low regulatory status and impartial regulatory data. In such cases, the formulator should emphasize the feasibility of formulation that can be approved for the market.

The emphasis must balance the concentration of additives along with the medical indication of the compound. Particularly the high concentrations of surface actives must be critically evaluated in view of risk/benefit aspects. The concentrations of surfactants and the additives should be optimised for achieving the maximum drug solubilization. From the solubility studies, it is probable that alone pure oils or a minimal surfactant concentration provide insufficient solvent capacity for a molecule. Apart from the aforesaid possibility of preferring SEDDS/SMEDDS over pure oils. Further SMEDDS are positively compared against type I systems over biopharmaceutical advantages. These self-emulsifying systems are believed to be less reliant on lipolysis than simple oil mixtures $[68,69]$. SMEDDS potentially lowers susceptibility to the discrepancy in exposure due to variable lipid digestion.

\section{Evaluation of phase behaviour and biological properties of excipients}

For an efficient lipid-based system needs a thorough knowledge of mixing behaviour of the components. Ternary phase diagrams are often employed to determine structural phase behaviour from subsequent emulsification and further characterizing behaviour of a formulation through dilution path $[70,71]$. An example represented in (fig. 2) dilution of the formulation is characterized from line A to B consisting primarily of $35 \%$ surfactant and $65 \%$ oil passing over regions of a $\mathrm{W} / 0$ microemulsion and a lamellar liquid crystal until attaining a stable bi-continuous $0 / \mathrm{W}$ microemulsion post-dilution. For a given formulation it is significant to understand the structures arising along dilution path to guarantee stable dispersed structures upon dilution. Optimum ratios of low and high HLB surfactants potentially yield smaller droplet size over single surfactant. Such more complex mixtures can be evaluated using pseudo-ternary diagrams where a specified surfactant mixture and oil mixture would aid as the oil or surfactant top of the diagram; likewise, cosolvents can be evaluated as part of the oil or surfactant mixture.

Preferably drug solubility and dispersions are evaluated simultaneously. Although the presence of co-solvent may improve drug solubility in the formulation system, high amounts of hydrophilic solvents result in drug precipitation post dispersion as a result of diffusion of co-solvent into the bulk aqueous phase. Presence of adequate amount of lipid aids in the formation of supersaturated systems that certainly lowers precipitation for a suitable period [72]. With recent high-throughput screening enables evaluating drug solubility and dispersion properties concurrently that assist in determining formulations with optimal behaviour conforming to both factors [73].

For the suggestion of candidate formulations further requires consideration of biological excipients effects. Mainly pertaining to drug efflux transporters and their modulation. Certain key aspects are discussed concerning SMEDDS formulations.

Most of the drugs are inherently substrates of efflux transporters that are positioned in the apical membrane of the human intestine. P-gp is known to possess a broad range of substrates few e. g. are doxorubicin, paclitaxel, saquinavir, cyclosporin, dexamethasone, and cimetidine [74-79]. A more recent defined ATP-binding cassette (ABC) transporter is BCRP. It is learned that it excretes mitoxantrone and further substrates were reported as prazosin, imatinib [80-81] furthermore, the $\mathrm{ABC}$ transporter that belongs to the group of multidrug resistance proteins like P-gp. Multidrug resistant-associated protein 2 (MRP2) also efflux for reported e. g. pravastatin, ritonavir certainly expressed on the apical side of enterocytes [82-84].

Lipid-based excipients are capable of interacting with efflux transporters and most significant with surface actives. Some of the 
reported surface actives are listed in (table 4) which exhibited drug transport inhibition. Majority of the orally accepted surface actives interact with either of the efflux transporters. Though the specificity makes a difference. Inhibition of P-gp was evident in many cases while interaction with BCRP was seen only with pluronic block polymers and polysorbate 20 . Such transport inhibition was not reported for polysorbate 80 , but both grades 20 and 80 would effectively impact MRP efflux.

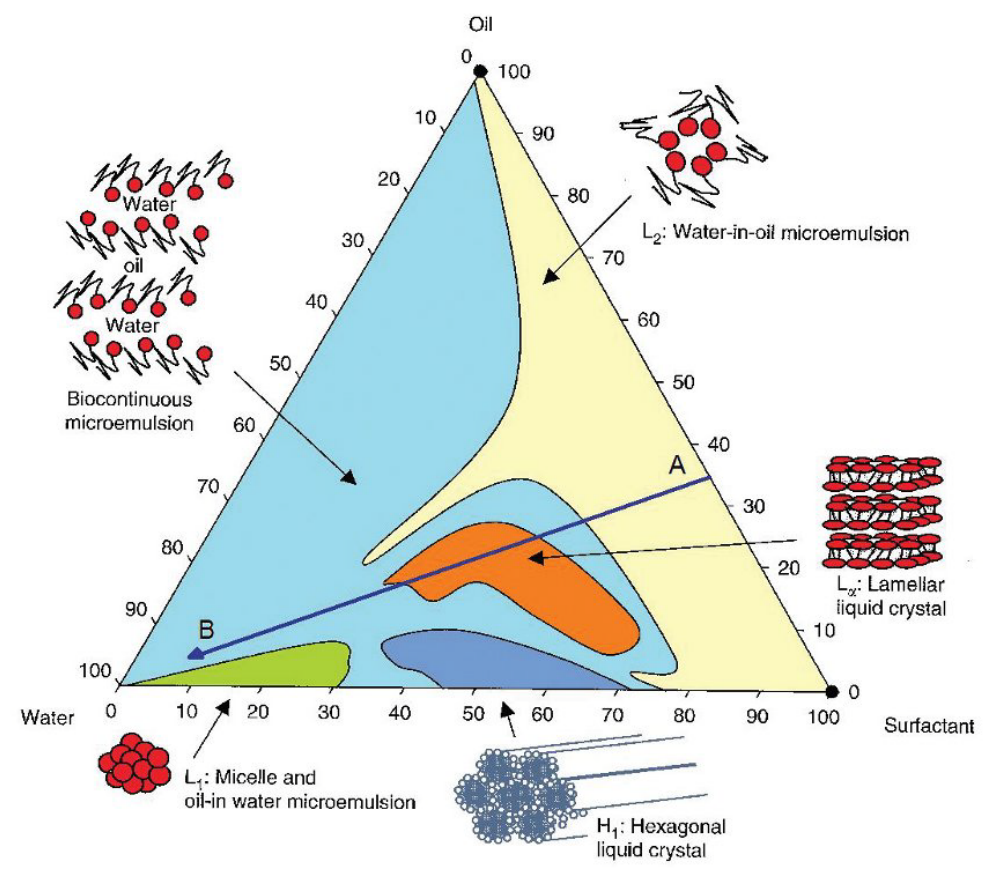

Fig. 2: Exemplary ternary phase diagram of an oil-surfactant water system, based on a C12E10-oleic acid water system [85]

The references mentioned in (table 4) were attained under diverse experimental conditions. Hence it is hard to relate these findings to human drug absorption. Though certain clinical absorption data is available results are difficult to interpret since several factors influence the fraction of drug absorbed. Differentiation of these bewildering effects, therefore, is a significant challenge. It is acknowledged from in vitro tests that P-gp inhibitors are frequently also inactivated cytochrome $\mathrm{P}_{450}$ (CYP)3A4 [86]. There is a large overlay of the inhibitor specificity of CYP3A4 and P-gp, as a result, drug efflux is not an isolated mechanism, but it is related to the extent of drug metabolism [87].

In an overview, excipients are scrutinized in regard to drug solvent capacity and phase behavior. If there is a rationale for suggested drug biological aspects along with effects of efflux transporters and CYP3A4 should be reviewed. After a thorough screening candidate system that exhibits encouraging results is proposed and further in vitro tests are performed. The significance of the aforementioned screening tests is debated matter hence it is judicious to have a better understanding at the level of absorption.

Table 4: List of surfactants that inhibit efflux transporters

\begin{tabular}{ll}
\hline Excipient & Transporter \\
\hline Polyoxyl 40 hydrogenated castor oil (Cremophor RH40) & P-gp \\
Polyoxyl 35 castor oil (Cremophor EL) & P-gp \\
Capryol caproyl macrogol glycerides (Labrasol) & P-gp \\
Medium-chain mono-di-and tri-glycerides (Imwitor 742) & P-gp \\
Lauryl macrogol-glycerides (Gelucire 44/14) & P-gp \\
A mixture of diacylglycerols (Peceol) & P-gp \\
Phospholipids & P-gp \\
Pluronic block copolymers (Pluronic P85) & P-gp, MRPs, BCRP \\
Polyoxyethylene sorbitan monooleate (Polysorbate 80) & P-gp, MRPs \\
Polyoxyethylene sorbitan monolaurate (Polysorbate 20) & P-gp, MRPs, BCRP \\
D- $\alpha$-Tocopheryl PEG 1000 succinate (TPGS) & P-gp \\
PEG 660-12-hydroxy-sterate (Solutol HS) & P-gp \\
\hline
\end{tabular}

\section{Characterization of lipid-based systems}

\section{In vitro studies}

The in vitro testing should mimic the environment of the stomach and intestine anticipate the performance. A primary understanding of formulation development and its drug release can be attained from in vitro testing. Studying the modifications of the formulation in the GIT is crucial for understanding drug absorption from lipid- based formulations. The release study from formulation system can foresee in vivo performance or can mainly aid as a quality control tool. The test technique and the medium are selected based on the objective. In common all tests pertain to the generation of new interfaces by dispersion. Droplets or colloidal structures produced upon dilution from which drug is released to acceptor medium [88].

Probably the $\mathrm{pH}$ influences the release rate from the formulation system. Certain drugs exhibit pH-dependent solubility moreover 
dispersion itself can be affected by $\mathrm{pH}$. Generation of new interfaces is greatly influenced by proton concentration or ionic strength besides the zeta potential of the particles. Mostly poorly soluble bases are sensitive to $\mathrm{pH}$. In this case, though a neutral to alkaline $\mathrm{pH}$ range is most crucial concerning low solubility. Drug precipitation is of more concern regarding lipid formulation, the kinetics and extent of drug precipitation is a measure for selecting one formulation over other. However, the in vivo condition should be precisely studied since the relevance of such invitro precipitation is specifically uncertain concerning drugs that are highly permeable. Rapid drug absorption may occur in vivo so that removal of luminal drug averts a crushing out. Such occurrences are not measured by a simple release, neither the digestion process is considered. The latter characteristic led to the development of a dynamic invitro lipolysis model.

Testing requires a pH-stat titration system; the basic working principle is maintaining a persistent $\mathrm{pH}(\mathrm{pH}$ must be maintained to avoid inhibition of the lipase) throughout a reaction which releases/consume hydrogen ions. Origination of any deviation is compensated by reagent addition. The model comprises of a temperature-controlled vessel $\left(37^{\circ} \mathrm{O}\right.$, which holds a suitable medium, composed of digestion buffer, phospholipid and bile salt. Calcium chloride may be primarily added or it can be continuously pumped throughout the process. Lipid-based formulation system is introduced into this model, and the digestion process is initiated further pancreatic lipase and co-lipase are added. Commencement of digestion results in the liberation of fatty acids, thereby a transient drop in $\mathrm{pH}$ is observed. This drop is measured by the $\mathrm{pH}$ electrode. The $\mathrm{pH}$ electrode is attached to a $\mathrm{pH}$-stat meter controller apart from auto-burette. An equimolar amount of sodium hydroxide is added to titrate the liberated fatty acids with the auto-burette, so as to avoid a variation in $\mathrm{pH}$ of digestion medium. The degree of digestion can be measured by quantifying the rate of addition of sodium hydroxide and considering the stoichiometric relation between fatty acids and sodium hydroxide. Throughout the digestion, process samples can be withdrawn and divided into the poorly dispersed oil phase, highly dispersed aqueous phase and precipitated pellet phase obtained by centrifugation. Computing amount of drug in the highly dispersed aqueous phase suggests drug has not precipitated, from which an assumption can be made concerning in vivo performance. The concentration of the bile salts, calcium and lipase activity had an influence on the initial rate of hydrolysis while the consequent stages are influenced by calcium concentration and lipase activity $[89,90]$. Dahan and Hoffman have presented the significance of in vitro lipolysis model for optimizing the oral-based formulation concerning to pre-systemic metabolism in the gut [91].

\section{In vivo studies}

Suitable in vivo studies significantly assess the influence of excipients pertaining bioavailability and pharmacokinetic profile of the drug. Subsequently, lipid-based formulations increase the bioavailability through improving the intestinal uptake hence a comprehensive understanding of the intestinal lymphatic absorption is required. As a reason of inadequate clinical data and variations in the method and animal model employed, it has become tough to carry studies involving the drug transport [92]. Henceforth work has to be carried out to establish an in vivo method and model to foresee lymphatic drug transport. A study in a mesenteric lymph ductcannulated rat model was performed to learn the mechanism for enhanced bioavailability for marketed formulation (Fortovase ${ }^{\circledR}$ ). The results revealed that the principal reason for improved bioavailability was due to enhanced solubilization and permeability of the drug in the lipid-rich pre-absorptive intestinal environment [93].

In lymphatic absorption, the significant step is the interaction of the drug with the chylomicrons in the enterocytes. To study the abovementioned phenomenon, an experimental rat model was used with blocked chylomicron flow to explicate the lymphatic transport of vitamin $\mathrm{D}_{3}$ was performed. When the results of mesenteric lymph duct-cannulated rat model were compared it exhibited that the association with chylomicrons guided a $75 \%$ of the vitamin $\mathrm{D}$ being absorbed through lymphatic uptake [94-96]. The influence of food (mostly high fatty meal) on the bioavailability of cyclosporin was considered by formulating the drug as Sandimmune Neoral ${ }^{\circledR}$ (Lipidbased system), and simple emulsion encapsulated in soft gelatin capsules and was subjected to healthy human volunteers. It was clear from the results that the lipid-based system is less affected by food when compared to the substantial effect of food from the simple emulsion. This significantly facilitated a broad range to the lipid-based system with respect to their dietary supplement.

\section{In vitro-in vivo correlation (IVIVC)}

Amidon et al. pivotal article describes the use of in vitro-in vivo correlation for various BCS classes. BCS class I drugs are the most suitable candidates while the class IV drugs are considered to be poor candidates for IVIVC due to their solubility and permeability characteristics. Whereas for class III IVIVC applicability is restricted due to their poor permeability. The solubility properties for class II compounds can critically improve with lipid-formulation thus achieving comparable to class I compounds. The rapid release profiles attained will allow the time required to be prominently less than gastric emptying time, thus improving the possibility of accomplishing robust IVIVC correlation [97]. Lipid-formulations belonging to type II and III rapidly disperse in the biological fluids thereby complete solubilization in biological fluids is achieved in lesser time which increases the potential for accomplishing IVIVC for class II compounds.

A comprehensive IVIVC will guide to develop potential and commercially viable lipid-system. A quick drug development with better product attribute could be accomplished with a potential model that correlates the in vitro and in vivo data. Computing the solubility, dissolution, intestinal membrane methods (cell culture and isolated animal tissue models) along with lipolysis of the lipid excipient are several in vitro techniques and are prominently employed to evaluate lipid-based formulations [98]. Aforesaid techniques suggest information limiting to specific aspects of the formulation. However, it is important to understand the in vivo interaction and performance of these systems.

Chylomicrons are produced and secreted by Caco- 2 cells on exposure to lipids, similar to in vivo enterocytes. The rat is a dependable model for preliminary screening and determining a formulation prototype. But due to diverse anatomical and physiological aspects of rat, like the absence of gallbladder and unalike expression and pattern levels for intestinal enzymes makes a correlation to human beings difficult. Several lipid-systems have been assessed employing dog model, however, poor correlation was observed amid dog and human due to variations in GIT physiology, like gastric $\mathrm{pH}$ besides enzymatic profile of enterocytes [99]. The pig deliberately believed to be a most suitable non-primate animal model for achieving close relation amongst the GIT anatomy and physiology [100]. This model allows the oral administration of the complete human dose, also food effects can be studies. The influence of intestinal absorption and first-pass metabolism of drugs can be studied with the aid of a pig model [101]. Therefore, to establish consistent in vitro-in vivo correlation in lipid-systems, ideal and most appropriate in vitro and in vivo models is of prime importance.

\section{Final dosage form besides comparison of several technological strategies}

Several prospects are available for developing the final dosage form of the lipid-based system for marketing. Modifications of the compositions can be considered only if the dosage form is categorised early enough. Characteristics regarding the filling process besides the compatibility of fill mass with capsule shells etc must be considered. Mostly process includes rotary die filling of soft gelatin capsules though other techniques have immensely gained importance over recent years.

Industries are currently keen in using alternative materials apart from gelatin for capsule shells. These days technical advancements enable to liquid-fill hard gelatin capsules on different batch scales. Sealing is achieved either by banding or by using liquid encapsulation by micro spray (LEMS), comparably it is less complicated to produce liquid-fill hard gelatin capsules than soft capsule technology [102]. Additionally, former resist much high 
filling temperatures $\left(\sim 70^{\circ} \mathrm{O}\right.$ compared to soft gelatin $\left(\sim 40^{\circ} \mathrm{O}\right.$. Excluding these benefits of hard gelatin, other aspects suggest the benefits of soft gelatin technology. Soft gelatin capsules have an edge for shell compatibility for hygroscopic excipients. Furthermore, an insufficient filling of the two-piece hard capsules can encourage leaking of fill mass. Most prominently more fill mass, and consequently higher doses can be subsumed into soft gelatin capsules since unlike hard gelatin capsules which possess headspace these are completely filled. To infer soft and hard capsules equally have merits and demerits so that no technology is basically considered superior over other. For specific projects necessity, suitable technology is selected. Right so for solid lipid-based systems. Various technologies exist for this formulation that suggests an alternative to the typical incorporation of a fill mass into a capsule.

Typically, lipid-based systems can be transformed into a solid dosage form is achieved by adsorbing onto a carrier [103-105] Nanoparticulate excipients are mostly preferred due to their high surface area as solid carriers. Adsorption onto the carriers is achieved certainly in a high shear mixer. As an alternative technique melt granulation is used with simple mixtures of waxy excipients with a traditional granulation process. This technique has an edge of producing high yielding drug load as the drug exists in coarse crystalline form. Adsorbates possess biopharmaceutical benefits of the larger surface area beside amorphous physical state. Solid dispersions by means of lipid-based excipients are highly commendable, owing to their high dose along with rapid release profile. But similar to other systems the solid dispersion must be evaluated for in-vitro to assess the potential of the drug to remain in solubilized form post-dilution. Furthermore, lipid excipients exhibit prominent mechanisms to improve bioavailability. Hence it is significant for the final dosage form to comprise high lipid content. High lipid load can simply attain by filling hard/soft gelatin capsules. These typically have potential scale-up properties unlike melt granulation or adsorbates. Albeit the latter techniques can deliver tablets and have benefits over capsules in terms of cost control [106]. All these signify that selection of dosage form must possess ideal biopharmaceutical aspects along with technical considerations besides economical. (table 5) enlists Food and Drug Administration (FDA) approved drugs employing lipid systems.

Table 5: FDA approved drugs employing lipid systems

\begin{tabular}{|c|c|c|c|c|}
\hline Molecule/Trade name & New drug application (NDA) year & LFCS & Drug category & References \\
\hline Ergocalciferol (Drisdol ${ }^{\circledR}$ ) & 1941 & I & Vitamin D analog & [107] \\
\hline Calcitriol (Rocaltrol ${ }^{\circledR}$ ) & 1978 & I & Vitamin D analog & [107] \\
\hline Valproic acid (Depakene ${ }^{\circledR}$ ) & 1978 & I & Anti-epileptic & {$[107]$} \\
\hline Isotretinoin (Accutane ${ }^{\circledR}$ )-discontinued & 1982 & I & Retinoid & [107] \\
\hline Cyclosporin A (Sandimmune ${ }^{\circledR}$ ) & 1983 & II & Calcineurin inhibitor & [107] \\
\hline Dronabinol (Marinol ${ }^{\circledR}$ ) & 1985 & I & Cannabinoid & [107] \\
\hline Clofazimine (Lamprene ${ }^{\circledR}$ )-discontinued & 1986 & I & Anti-leprosy & [107] \\
\hline Cyclosporin A (Sandimmune ${ }^{\circledR}$ ) & 1990 & II & Calcineurin inhibitor & [107] \\
\hline Ranitidine (Zantac ${ }^{\circledR}$ )-discontinued & 1994 & & H2-recptor antagonist & [107] \\
\hline Cyclosporin A (Neoral ${ }^{\circledR}$ ) & 1995 & IIA/IIIB & Calcineurin inhibitor & [107] \\
\hline Cyclosporin A (Neoral ${ }^{\circledR}$ ) & 1995 & IIA/IIIB & Calcineurin inhibitor & [107] \\
\hline Tretinoin (Vesanoid ${ }^{\circledR}$ )-discontinued & 1995 & I & Retinoid & [107] \\
\hline Ritonavir (Norvir ${ }^{\circledR}$ ) & 1996 & IIIA & Protease inhibitor & [107] \\
\hline Saquinavir (Fortovase ${ }^{\circledR}$ )-discontinued & 1997 & & Protease inhibitor & [107] \\
\hline Progesterone (Prometrium ${ }^{\circledR}$ ) & 1998 & I (susp) & Hormone & [107] \\
\hline $\begin{array}{l}\text { Amprenavir (Agenerase }{ }^{\circledR} \text { )- } \\
\text { discontinued }\end{array}$ & 1999 & IV & Protease inhibitor & {$[107]$} \\
\hline Bexarotene (Targretin ${ }^{\circledR}$ ) & 1999 & IV & Retinoid & [107] \\
\hline Doxecalciferol (Hectorol ${ }^{\circledR}$ ) & 1999 & I & Vitamin D analog & [107] \\
\hline Sirolimus (Rapamune ${ }^{\circledR}$ ) & 1999 & III & mTOR kinase inhibitor & [107] \\
\hline Cyclosporin A (Gengraf ${ }^{\circledR}$ ) & 2000 & IV & Calcineurin inhibitor & [107] \\
\hline Cyclosporin A (Gengraf ${ }^{\circledR}$ ) & 2000 & IV & Calcineurin inhibitor & [107] \\
\hline $\begin{array}{l}\left.\text { Ritonavir/lopinavir (Kaletra }{ }^{\circledR}\right)- \\
\text { Discontinued }\end{array}$ & 2000 & III & Protease inhibitor & [107] \\
\hline Dutasteride (Avodart ${ }^{\circledR}$ ) & 2001 & I & 5 alpha-reductase inhibitor & [107] \\
\hline Isotretinoin (Claravis ${ }^{\circledR}$ ) & 2003 (ANDA) & & Retinoid & [107] \\
\hline Omega-3-acid ethyl esters (Lovaza ${ }^{\circledR}$ ) & 2004 & I & Fish oil for lipid digestion & [107] \\
\hline Tipranavir (Aptivus ${ }^{\circledR}$ ) & 2005 & IIIA & Protease inhibitor & [107] \\
\hline Tipranavir (Aptivus ${ }^{\circledR}$ ) & 2005 & IV & Protease inhibitor & [107] \\
\hline Paricalcitol (Zemplar ${ }^{\circledR}$ ) & 2005 & $\mathrm{I}$ & Vitamin D analog & [107] \\
\hline Lubiprostone (Amitiza ${ }^{\circledR}$ ) & 2006 & I & Chloride channel activator & [107] \\
\hline Fenofibrate (Lipofen ${ }^{\circledR}$ ) & 2006 & III & $\begin{array}{l}\text { Peroxisome proliferator } \\
\text { activated receptor } \alpha \text { (PPAR } \alpha) \\
\text { activator }\end{array}$ & {$[107]$} \\
\hline Topotecan HCl (Hycamtin ${ }^{\circledR}$ ) & 2007 & I & Topoisomerase inhibitor & [107] \\
\hline Loratadine (Claritin ${ }^{\circledR}$ ) & 2008 & & Antihistamine & [107] \\
\hline Isotretinoin (Absorica ${ }^{\circledR}$ ) & 2012 & & Retinoid & [107] \\
\hline Enzalutamide (Xtandi ${ }^{\circledR}$ ) & 2012 & I & Androgen receptor inhibitor & [107] \\
\hline Nintedanib $\left(\mathrm{Ofev}^{\circledR}\right)$ & 2014 & II & Tyrosine kinase inhibitor & [107] \\
\hline Calcifediol (Rayaldee ${ }^{\mathrm{TM}}$ ) & 2016 & II/III & Vitamin D analog & [107] \\
\hline
\end{tabular}

\section{CONCLUSION}

A significant opportunity along with a potential challenge is anticipated from most insoluble drug molecules approved by FDA, besides those in the development stage. At present lipid-systems are in emphasis for developing formulation of poorly water-soluble drugs. Self-emulsifying systems deliver an appropriate approach to deal with a biopharmaceutical challenging drug. To understand a clear rationale for the formulation development, precise biopharmaceutical aspects should be considered. Later a thorough sequence of screening tests is performed for the selection of formulation excipients. The crucial intent is to retain the drug in solubilized form since drug precipitation confines the oral bioavailability. The portion of this preliminary focus on drug 
absorption is the consideration of excipient effects on presystemic clearance or inhibition of efflux transporters. Prior to the final composition certain technical aspects are considered pertaining to product manufacture. The excipient concentration and their grades can only be altered within the parameters of solubility and phase behaviour. With specific rationale, it seems worth capitalizing development resources in lipid-based systems. Given the major biopharmaceutical potential it is believed that more new actives will be formulated as lipid-systems, also will benefit from this fascinating formulation approach.

\section{AUTHORS CONTRIBUTIONS}

All the authors have contributed equally

\section{CONFLICTS OF INTERESTS}

All authors have none to declare

\section{REFERENCES}

1. Tang B, Cheng G, Gu JC, Xu CH. Development of solid selfemulsifying drug delivery system; preparation techniques and dosage forms. Drug Discovery Today 2008;13:606-12.

2. Yao G, Li Y. Preparation, characterization and evaluation of selfmicro emulsifying drug delivery systems (SMEDDSs) of ligusticum chuanxiong oil. Biomed Prev Nutr 2010;1:36-42.

3. Amri A, Clanche SL, Therond P, Bonnefont Rousselot D, Borderie D, Lai-Kuen $\mathrm{R}$, et al. Resveratrol self-emulsifying system increases the uptake by endothelial cells and improves protection against oxidative stress-mediated death. Eur J Pharm Biopharm 2013;86:418-26.

4. Pund S, Shete Y, Jagdale S. Multivariate analysis of physicochemical characteristics of lipid-based nano emulsifying cilostazol-quality by design. Colloids Surf B 2014;115:29-36.

5. Pouton CW. Formulation of poorly water-soluble drugs for oral administration: physicochemical and physiological issues and the lipid formulation classification system. Eur J Pharm Sci 2006;29:278-87.

6. Hauss DJ. Oral lipid-based formulations. Adv Drug Delivery Rev 2007;59:667-76.

7. Nanjwade B, Patel DJ, Udhani R, Manvi F. Functions of lipids for enhancement of oral bioavailability of poorly water-soluble drugs. Sci Pharm 2011;879:705-27.

8. Mullertz A, Ogbonnaa A, Ren S, Rades T. New perspectives on lipid and surfactant based drug delivery systems for oral delivery of poorly soluble drugs. J Pharm Pharmcol 2010;62:1622-36.

9. Singh AK, Chaurasiya A, Singh M, Upadhyay SC, Mukherjee R, Khar RK. Exemestane loaded self-micro emulsifying drug delivery system (SMEDDS) development and optimization. AAPS PharmSciTech 2008;9:628-34.

10. Singh B, Bandopadhyay S, Kapil R, Singh R, Katare O. Self emulsifying drug delivery systems (SEDDS): formulation development, characterization, and applications. Crit Rev Ther Drug Carrier Syst 2009;26:427-1.

11. Zidan AS, Sammour OA, Hammad MA, Megrab NA, Habib MJ, Khan MA. Quality by design: understanding the product variability of a self-nanoemulsified drug delivery system of cyclosporine A. J Pharm Sci 2007;96:2409-23.

12. Wang Z, Sun J, Wang Y, Liu X, Liu Y, Fu Q, et al. Solid selfemulsifying nitrendipine pellets: preparation and in vitro/in vivo evaluation. Int J Pharm 2010;38:1-6.

13. Pouton CW. Lipid formulations for oral administration of drugs: non-emulsifying, self-emulsifying, and self-micro emulsifying drug delivery systems. Eur J Pharm Sci 2000;11:S93-8.

14. Gursoy RN, Benita S. Self-emulsifying drug delivery systems (SEDDS) for improved oral delivery of lipophilic drugs. Biomed Pharmacother 2004;58:173-82.

15. Khan AW, Kotta S, Ansari SH, Sharma RK, Ali J. Potentials and challenges in self-nano emulsifying drug delivery systems. Expert Opin Drug Delivery 2012;9:1305-17.

16. Mahmoud EA, Bendas ER, Mohamed MI. Preparation and evaluation of self-nano emulsifying tablets of carvedilol. AAPS PharmSciTech 2009;10:183-92.
17. Gao P, Morozowich W. Development of super saturable selfemulsifying drug delivery system formulations for improving the oral absorption of poorly soluble drugs. Expert Opin Drug Delivery 2006;3:97-110.

18. Sonia Anand, Rishikesh Gupta, Prajapati SK. Self-micro emulsifying drug delivery system. Asian J Pharm Clin Res 2016;9:33-8.

19. Taha E, Ghorab D, Zaghloul AA. Bioavailability assessment of vitamin a self-nanoemulsified drug delivery systems in rats: a comparative study. Med Princ Pract 2007;16:355-9.

20. Sander C, Holm P. Porous magnesium aluminometasilicate tablets as a carrier of a cyclosporine self-emulsifying formulation. AAPS PharmSciTech 2009;10:1388-95.

21. Porter $\mathrm{CJH}$, Trevaskis NL, Charman WN. Lipids and lipid-based formulations: optimizing the oral delivery of lipophilic drugs. Nat Rev Drug Discovery 2007;6:231-8.

22. Bowtle W. Materials, process, and manufacturing considerations for lipid-based hard-capsule formats. In: Hauss DJ. editor. Oral lipid-based formulations are enhancing the bioavailability of poorly water-soluble drugs. 1st ed. Vol. 170. New York: Informa Healthcare; 2010. p. 79-106.

23. Trevaskis NL, Charman WN, Porter CJH. Lipid-based delivery systems and intestinal lymphatic drug transport: a mechanistic update. Adv Drug Delivery Rev 2008;60:702-16.

24. Zhang P, Liu Y, Xu J. Preparation and evaluation of selfemulsifying drug delivery system of oridonin. Int J Pharm 2008;355:269-76.

25. Wasylaschuk WR, Harmon PA, Wagner G. Evaluation of hydroperoxides in common pharmaceutical excipients. J Pharm Sci 2007;96:106-16.

26. Driscoll CMO. Lipid-based formulations for intestinal lymphatic delivery. Eur J Pharm Sci 2002;15:405-15.

27. Porter CJH, Trevaskis NL, Charman WN. Lipids and lipid-based formulations: optimizing the oral delivery of lipophilic drugs. Nat Rev Drug Discovery 2007;6:231-48.

28. Charman WN, Porter CJ, Mithani S, Dressman JB. Physiochemical and physiological mechanisms for the effects of food on drug absorption: the role of lipids and pH. J Pharm Sci 1997;86:269-82.

29. Humberstone AJ, Charman WN. Lipid-based vehicles for the oral delivery of poorly water-soluble drugs. Adv Drug Delivery Rev 1997;25:103-28.

30. Pramod K, Peeyush K, Rajeev K, Nitish K, Rakesh K. An overview of lipid-based formulation for oral drug delivery. Drug Inv Today 2010;2:390-5.

31. Erlanson Albertsson C. Pancreatic colipase. Structural and physiological aspects. Biochim Biophys Acta 1992;1125:1-7.

32. Van den Bosch H, Postema NM, de Haas GH, van Deenen L. On the positional specificity of phospholipase a from the pancreas. Biochim Biophys Acta 1965;98:657-9.

33. Pouton CW, Porter CJH. Formulation of lipid-based delivery systems for oral administration: materials, methods and strategies. Adv Drug Delivery Rev 2008;60:625-37.

34. Humberstone AJ, Charman WN. Lipid-based vehicles for the oral delivery of poorly water-soluble drugs. Adv Drug Delivery Rev 1997;25:103-28.

35. Porter $\mathrm{CJ} \mathrm{H}$, Charman $\mathrm{WN}$. Uptake of drugs into intestinal lymhatics after oral administration. Adv Drug Delivery Rev 1997;25:71-89.

36. Pouton CW. SEDDS: assessment of the efficiency of the emulsion. Int J Pharm 1985;27:335-48.

37. Reiss H. Entropy induced dispersion of bulk liquids. J Colloid Interface Sci 1975;53:61-70.

38. Dabros T, Yeung A, Masliyah J, Czarnecki J. Emulsification through area contraction. J Colloid Interface Sci 1999;210:222-4.

39. Wakerly MG, Pouton CW, Meakin B, Morton F. Self emulsification of veg: oil-nonionic-surfactant mixtures. ACS Symp Ser 1986;311:242-55.

40. Wakerly MG, Pouton CW, Meakin BJ. Evaluation of the selfemulsifying performance of a non-ionic surfactant vegetable oil mixture. J Pharm Pharmacol 1987;39:6.

41. Gao P, Morozowich W. Improving the oral absorption of poorly soluble drug using SEDDS and S-SEDDS formulations. In: Qiu Y, Chen Y, Zhang GGZ. editors. Developing solid oral dosage forms 
theory and practice. 2nd ed. New York: Academic Press; 2009. p. 443-9.

42. Land LM, Li P, Bummer PM. The influence of water content of triglyceride oils on the solubility of steroids. Pharm Res 2005;22:784-8

43. Hauss DJ. Oral lipid-based formulations. Adv Drug Delivery Rev 2007;59:667-76.

44. Azaz E, Donbrow M, Hamburger R. Incompatibility of non-ionic surfactants with oxidisable drugs. Pharm J 1973;211:15.

45. Carlert S, Palsson A, Hanisch G, Von Corswant C, Nilsson C, Lindfors $\mathrm{L}$, et al. Predicting intestinal precipitation-a case example for a basic BCS class II drug. Pharm Res 2010;27:2119-30.

46. Rahman MA, Hussain A, Hussain MS, Mirza MA, Iqbal Z. Role of excipients in the successful development of selfemulsifying/micro-emulsifying drug delivery system (SEDDS/SMEDDS). Drug Dev Ind Pharm 2013;39:1-19.

47. Cuine JF, Mc Evoy CL, Charman WN, Pouton CW, Edwards GA, Benameur $\mathrm{H}$, et al. Evaluation of the impact of surfactant digestion on the bioavailability of danazol after oral administration of lipidic self-emulsifying formulations to dogs. J Pharm Sci 2008;97:995-1012.

48. Pawar S, Kumar A. Issues in the formulation of drugs for oral use in children. Pediatr Drugs 2002;4:371-9.

49. Lopez Montilla JC, Herrera Morales PE, Pandey S, Shah DO. Spontaneous emulsification: mechanisms, physicochemical aspects, modeling, and applications. J Dispers Sci Technol 2002;23:219-68.

50. Basit AW, Podczeck F, Newton JM, Waddington WA, Ell PJ, Lacey LF. Influence of polyethylene glycol 400 on the gastrointestinal absorption of ranitidine. Pharm Res 2002;19:1368-74.

51. Schulze JDR, Waddington WA, Ell PJ, Parsons GE, Coffin MD, Basit AW. Concentration-dependent effects of polyethylene glycol 400 on gastrointestinal transit and drug absorption. Pharm Res 2003;20:1984-8.

52. Amidon G, Lennernas H, Shah V, Crison J. A theoretical basisfor a biopharmaceutical drug classification: the correlation of in vitro drug product dissolution and in vivo bioavailability. Pharm Res 1995;12:413-20.

53. Dressman J, Amidon G, Fleisher D. Absorption potential: estimation of the fraction absorbed for orally administered compounds. J Pharm Sci 1985;74:588-9.

54. Johnson K, Swindell A. Guidance in the setting of drug particle size specifications to minimize variability in absorption. Pharm Res 1996;13:1795-8.

55. Agoram B, Woltosz W, Bolger M. Predicting the impact of physiological and biochemical processes on oral drug bioavailability. Adv Drug Delivery Rev 2001;50:S41-67.

56. Willmann S, Schmitt W, Keldenich J, Lippert J, Dressman J. A physiological model for the estimation of the fraction dose absorbed in humans. J Med Chem 2004;47:4022-31.

57. Jamei M, Turner D, Yang J, Neuhoff S, Polak S, Rostami Hodjegan A, et al. Population-based mechanistic prediction of oral drug absorption. AAPS J 2009;11:225-37.

58. Kuentz M. Drug absorption modeling as a tool to define the strategy in clinical formulation development. AAPS J 2008;10:473-9.

59. Kuentz M, Nick S, Parrott N, Roethlisberger D. A strategy for preclinical formulation development using gastro plus as pharmacokinetic simulation tool and a statistical screening design applied to a dog study. Eur J Pharm Sci 2006;27:91-9.

60. Wasswik CM, Holmen AG, Artursson P, Bergström CAS. Molecular characteristics for solid-state limited solubility. J Med Chem 2008;51:3035-9.

61. Jill B Shukla, Girish K Jani, Abdel Wahab Omri. Formulation and evaluation of oral self micro emulsifying drug delivery system of candesartan cilexetil. Int J Pharm Pharm Sci 2016;8:238-43.

62. GB Preethi, HN Shivakumar, M Ravi Kumar, N Sweta. Prototype self-emulsifying system of etravirine: design, formulation and in vitro evaluation. Int J Appl Pharm 2018;10:13-9.

63. Mette G, Anette M. Liquid self-micro emulsifying drug delivery systems. In: David JH. editor. Oral lipid-based formulations. Special ed. New Jersey: Informa healthcare; 2007. p. 107-27.

64. Padley FB, Gunstone FD, HarwoodJ L. Occurrence and characteristics of oils and fats. In: Gunstone FD, Harwood JL,
Padley FB. editors. The lipid handbook. 2nd ed. London: Chapman and Hall; 1994. p. 47-224.

65. Hong JY, Kim JK, Song YK, Park JS, Kim CK. A new self-emulsifying formulation of itraconazole with improved dissolution and oral absorption. J Controlled Release 2006;110:332-8.

66. Patel AR, Vavia PR. Preparation and in vivo evaluation of SMEDDS (self-micro emulsifying drug delivery system) containing fenofibrate. AAPS J 2007;9:E344-52.

67. Swenson ES, Milisen WB, Curatolo W. Intestinal permeability enhancement: efficacy, acute local toxicity, and reversibility. Pharm Res 1994;11:1132-42.

68. Meinzer A, Mueller EA, Niese D, Choc G, Wong R, Vonderscher J, et al. Improved oral absorption of cyclosporine by using Neoral, a microemulsion formulation. In: Lieberman R, Mukherjee A. editors. Principles of Drug Development in Transplantation and Autoimmunity. Austin: RG Landes Company; 1996. p. 259-67.

69. Kuentz M, Wyttenbach N, Kuhlmann O. Application of a statistical method to the absorption of a new model drug from micellar and lipid formulations-evaluation of qualitative excipient effects. Pharm Dev Technol 2007;12:275-83.

70. Farah N, Laforet JP, Denis J. Self-micro emulsifying drug delivery systems (SMEDDS) for improving dissolution and availability of poorly soluble actives ingredients. BT Gattefosse 1992;87:41-7.

71. Constantinides PP, Welzel G, Ellens H, Smith PL, Sturgis SS, Yiv $\mathrm{SH}$, et al. Water-in-oil microemulsions containing mediumchain fatty acids/salts: formulation and intestinal absorption evaluation. Pharm Res 1996;13:210-5.

72. Mohsin K, Long MA, Pouton CW. Design of lipid-based formulations for oral administration of poorly water-soluble drugs: precipitation of drug after dispersion of formulations in aqueous solution. J Pharm Sci 2009;98:3582-95.

73. Cui J, Yu B, Zhao Y, Zhu W, Li H, Lou H, et al. Enhancement of oral absorption of curcumin by self-micro emulsifying drug delivery systems. Int J Pharm 2009;371:148-55.

74. Troutman MD, Thakker DR. Novel experimental parameters to quantify the modulation of absorptive and secretory transporter of compounds by P-glycoprotein in cell culture models of intestinal epithelium. Pharm Res 2003;20:1210-24.

75. Collet A, Tanianis Hughes J, Hallifax D, Warhurst G. Predicting P-glycoprotein effects on absorption: correlation of transport in caco-2 with drug pharmacokinetics in wild-type and mdr1a (-/-) mice in vivo. Pharm Res 2004;21:819-26.

76. Kim RB, Fromm MF, Wandel C, Leake B, Wood AJ, Roden DM, et $a l$. The drug transporter P-glycoprotein limits oral absorption and brain entry of HIV-1 protease inhibitors. J Clin Invest 1998;101:289-94.

77. Saeki T, Ueda K, Tanigawara Y, Hori R, Komano T. Human Pglycoprotein transports cyclosporin A and FK506. J Biol Chem 1993;268:6077-80.

78. Ueda K, Okamura N, Hirai M, Tanigawara Y, Saeki T, Kioka N, et al Human P-glycoprotein transports cortisol, aldosterone, and dexamethasone, but not progesterone. J Biol Chem 1992;267:24248-52.

79. Kim WY, Benet LZ. P-glycoprotein (P-gp/MDR1)-mediated efflux of sex-steroid hormones and modulation of P-gp expression in vitro. Pharm Res 2004;21:1284-93.

80. Litman T, Brangi M, Hudson E, Fetsch P, Abati A, Ross DD, et al. The multidrug-resistant phenotype associated with overexpression of the new ABC half-transporter, MXR (ABCG2) J Cell Sci 2000;113:2011-21.

81. Burger H, Van Tol H, Boersma AW, Brok M, Wiemer EA, Stoter $\mathrm{G}$, et al. Imatinib mesylate (STI571) is a substrate for the breast cancer resistance protein (BCRP)/ABCG2 drug pump. Blood 2004;104:2940-2.

82. Sasaki M, Suzuki H, Ito K, Abe T, Sugiyama Y. Transcellular transport of organic anions across a double-transfected madindarby canine kidney II cell monolayer expressing both human organic anion-transporting polypeptide (OATP2/SLC21A6) and multidrug resistance-associated protein 2 (MRP2/ABCC2). J Biol Chem 2002;277:6497-503.

83. Huisman MT, Smit JW, Crommentuyn KM, Zelcer N, Wiltshire $\mathrm{HR}$, Beijnen JH, et al. Multidrug resistance protein 2 (MRP2) 
transports HIV protease inhibitors, and transport can be enhanced by other drugs. AIDS 2002;16:2295-301.

84. Guo A, Marinaro W, Hu P, Sinko PJ. Delineating the contribution of secretory transporters in the efflux of etoposideusing MadinDarby canine kidney (MDCK) cells overexpressing Pglycoprotein (Pgp), multidrug resistance-associated protein (MRP1), and canalicular multispecific organic anion transporter (cMOAT). Drug Metab Dispos 2002;30:457-63.

85. Cannon JB, Long MA. Emulsions, microemulsions, and lipidbased drug delivery systems for drug solubilization and delivery, Part II: oral applications. In: Liu R. editor. Waterinsoluble drug formulation. 2nd ed. Boca Raton FL: CRC Press; 2008. p. 227-54

86. Zhou S, Chan E, Lee YL, Boelsterli UA, Li SC, Wang J, et al. Therapeutic drugs that behaveasmechanism-based inhibitors of cytochrome P450 3A4. A Curr Drug Metab 2004;5:415-42.

87. Hochman JH, Chiba M, Nishime J, Yamazaki M, Lin JH. Influence of P-glycoprotein on the transport and metabolism of indinavir in caco-2 cells expressing cytochrome P-450 3A4. J Pharmacol Exp Ther 2000;292:310-8.

88. Shah NH, Carvajal MT, Patel CI, Infeld MH, Malick AW. Selfemulsifying drug delivery systems (SEDDS) with polyglycolyzed glycerides for improving in vitro dissolution and oral absorption of lipophilic drugs. Int J Pharm 1994;106:15-23.

89. Zangenberg NH, Mullertz A, Kristensen HG, Hovgaard L. A dynamic in vitro lipolysis model I. Controlling the rate of lipolysis by continuous addition of calcium. Eur J Pharm Sci 2001;14:115-22.

90. Zangenberg NH, Mullertz A, Kristensen HG, Hovgaard L. A dynamic in vitro lipolysis model II: evaluation of the model. Eur J Pharm Sci 2001;14:237-44.

91. Dahan A, Hoffman A. Use of a dynamic in vitro lipolysis model to rationalize oral formulation development for poor water-soluble drugs: correlation with in vivo data and the relationship to intraenterocyte processes in rats. Pharm Res 2006;23:2165-74.

92. Edwards GA, Porter CJH, Caliph SM, Khoo SM, Charman WN. Animal models for the study of intestinal lymphatic drug transport. Adv Drug Delivery Rev 2001;50:45-60.

93. Griffin BT, O'Driscoll CM. A comparison of intestinal lymphatic transport and systemic bioavailability of saquinavir from three lipid-based formulations in the anaesthetized rat model. J Pharm Pharmacol 2006;58:917-25.

94. Dahan A, Hoffman A. Enhanced gastrointestinal absorption of lipophilic drugs. In: Touitou E, Barry BW. editors. Enhancement in drug delivery. 1st ed. Florida: CRC Press; 2006. p. 111-27.

95. Mueller EA, Kovarik JM, Bree JBV, Grevel J, Lucker PW, Kutz K, et al. Influences of a fat-rich meal on the pharmacokinetics of a new oral formulation of cyclosporine in a crossover comparison with the market formulation. Pharm Res 1994;11:151-5.

96. Dahan A, Hoffman A. The effect of different lipid-based formulations on the oral absorption of lipophilic drugs: the ability of in vitro lipolysis and consecutive ex vivo intestinal permeability data to predict in vivo bioavailability in rats. Eur J Pharm Biopharm 2007;67:96-105.

97. Dressman JB, Reppas C. In vitro in vivo correlations for lipophilic, poorly water-soluble drugs. Eur J Pharm Sci 2000;11 Suppl 2:S73-80.

98. Seeballuck F, Ashford MB, Driscoll CMO. The effects of pluronic block copolymers and cremophor EL on intestinal lipoprotein processing and the potential link with p-glycoprotein in caco-2 cells. Pharm Res 2003;20:1085-92.

99. Khoo SM, Shackleford DM, Porter CJH, Edwards GA, Charman WN. Intestinal lymphatic transport of halofantrine occurs after oral administration of a unit-dose lipid-based formulation to fasted dogs. Pharm Res 2003;20:1460-5.

100. Petri N, Bergman E, Forsell P, Hedeland M, Bondesson U, Knutson L, et al. First-pass effects of verapamil on the intestinal absorption and liver disposition of fexofenadine in the porcine model. Drug Metab Dispos 2006;3:1182-9.

101. Kararli TT. Comparison of the gastrointestinal anatomy, physiology, and biochemistry of humans and commonly used laboratory animals. Biopharm Drug Dispos 1995;16:351-80.

102. Cole ET, Cade D, Benameur H. Challenges and opportunities in the encapsulation of liquid and semi-solid formulations into capsules for oral administration. Adv Drug Delivery Rev 2008;60:747-56.

103. Ito Y, Arai H, Uchino K, Iwasaki K, Shibata N, Takada K. Effects of adsorbents on the absorption of lansoprazole with a surfactant. Int J Pharm 2005;289:69-77.

104. Madhavi K, Shikha A, Suresh B. Formulation and in vitro characterization solid self-emulsifying drug delivery system of ramipril prepared by adsorption technique. Int J Curr Pharm Res 2016;8:40-5.

105. Shweta K, Yogesh P. Formulation and evaluation of neusilin us2 adsorbed amorphous solid self-micro emulsifying delivery system of atorvastatin calcium $^{\circledR}$. Asian J Pharm Clin Res 2016;9:93-100.

106. Naser MY Hasan, Dhaifallah M Almalki, Mohammed JK Althuwaybi, Hassan M Alshehri. SMEDDS tablet: compatibility of solid SMEDDS using various pharmaceutical tablet excipients. Int J Pharm Pharm Sci 2016;8:246-51.

107. Ronak Savla, Jeff Browne, Vincent Plassat, Kishor M Wasan, Ellen $\mathrm{K}$ Wasan. Review and analysis of FDA approved drugs using lipid-based formulations. Drug Dev Ind Pharm 2017;43:1743-58. 